\title{
Automated Analysis of Remyelination Therapy for Spinal Cord Injury
}

\author{
Koel Das ${ }^{*}$, Aditi Majumder, Monica Siegenthaler ${ }^{\dagger}$, Hans Keirstead and M Gopi \\ University of California, Irvine, CA, USA \\ das@psych.ucsb.edu,majumder@ics.uci.edu,msiegent@uci.edu,hansk@uci.edu,gopi@ics.uci.edu
}

\begin{abstract}
Demyelination- the loss of myelin sheath that insulates axons, is a prominent feature in many neurological disorders resulting in spinal cord injury (SCI). The lost myelin sheath can be replaced by remyelination, used in SCI treatment. In this paper, we propose an algorithm for efficient automated analysis of remyelination therapy. We use a robust, shape-independent algorithm based on iso-contour analysis of the image at progressively increasing intensity levels for detecting cell boundaries. The detected boundaries of spatially clustered cells are then separated using Delaunay triangulation based contour separation method. The therapeutically important oligodendrocyte-remyelinated axons (OR-axons) are identified and a density map is generated for efficacy analysis of the therapy. Our efficient automated remyelination analysis significantly reduces error due to human subjectivity. We corroborate the accuracy of our results by extensive cross-verification by the domain experts.
\end{abstract}

\section{INTRODUCTION}

Demyelination - the loss of myelin sheath - is a prominent feature in many neurological disorders including multiple sclerosis (MS) and spinal cord injury (SCI) [13] which results in the disruption of signals within the axons. The lost myelin sheath can be replaced by a process called remyelination which wraps myelin sheath around the demyelinated axons restoring the conduction of signals within the axons. Remyelination can be achieved by two kinds of cells, oligodendrocytes and Schwann cells [3]. One of the many therapies being developed to improve the remyelination include transplantation of oligodendrocyte progenitor cells into the adult spinal cord of rats following SCI which helps in remyelination of the demyelinated axons. However, to study the progress of the therapy these oligodendrocyte-remyelinated axons (OR-axons), created due to oligodendrocyte progenitor cell transplantation, need to be distinguished from the already myelinated axons or axons remylinated by the Schwann cells already existing in the body. The OR-axons are identified by their characteristically thin myelin

*Corresponding author, Currently at UC Santa Barbara

${ }^{\dagger}$ Currently at California Stem Cell, CA,USA

Permission to make digital or hard copies of all or part of this work for personal or classroom use is granted without fee provided that copies are not made or distributed for profit or commercial advantage and that copies bear this notice and the full citation on the first page. To copy otherwise, to republish, to post on servers or to redistribute to lists, requires prior specific permission and/or a fee.

ICVGIP' 10, December 12-15, 2010, Chennai, India

Copyright 2010 ACM 978-1-4503-0060-5/10/12 ...\$10.00. sheaths relative to the diameter of the axons. This ratio of myelin sheath thickness to axon diameter is called the G-ratio [15]. Following the identification of OR-axons, their distribution has to be analyzed by visualizing the size, location and density of its clusters in the image. By tracking such visualizations of microscopic images periodically captured on different rat subjects before and after the treatment, one can understand the growth rate and site of the OR-axons and hence the effectiveness of the therapy.

\subsection{Motivation}

Remyelination is analyzed on microscopic images of $5 \times 625 \mu \mathrm{m}^{2}$ areas aligned on a radial oriented line that originates from the central canal of the spinal cord and extends to the outermost limit of the spinal cord cross section. The current method for identifying remyelinated axons is the line sampling technique [4] that is achieved manually. There are two kinds of error introduced in manually estimating and classifying the remyelinated axons. Since a manual process is very tedious, the OR-axons are only identified in approximately $15 \%$ of the actual area of pathology. In the rest of the area, it is only estimated. This leaves out a high percentage of area in which a large amount of estimation error can be introduced in the data. Further, there is classification error because of the subjectivity involved in the manual classification of the OR-axons. The G-ratio, which can be used to classify OR-axons, is not calculated for every single axon because this would be tedious and time intensive work. It is often assigned by relative measure through visual estimation. This again leads to more classification errors. It is not known how much error is introduced to the data when there are multiple examiners counting these axons. These errors can be reduced by increasing the percentage of the actual area of pathology, but the sheer size of the number of samples collected from the pathology makes this a daunting task. For example, since a microscopic slice is taken every $2 \mathrm{~mm}$ of the spinal cord of an animal, in experiments involving many animals, the OR-axon growth analysis can take several weeks. An increase in percentage of counted area would not only increase the analysis time, but might also increase the error due to human factors such as fatigue.

An automated method to analyze and visualize the distribution of the OR-axons can alleviate all the above problems and provide an incredibly useful tool for research in these kinds of cutting edge therapies. An automated system will allow analysis of a higher percentage of area of the pathology, at more frequent intervals, and at higher accuracy due to less subjectivity and human factor related errors. Thus, it is evident that automated recognition and classification of the axons would be imminent in reducing the turn-aroundtime and the subjective error in this research.

\subsection{Technical Challenges}

Automating the process of analysis and visualization of distribu- 

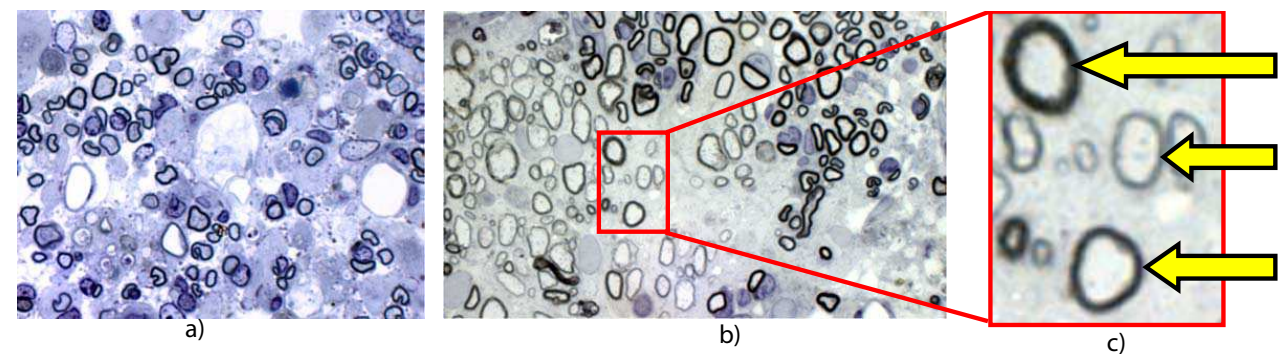

Normally

remyelinated axons

Oligodendrocyte-

remyelinated axons

Schwann cell-

remyelinated axons

Figure 1: Different kinds of cells in the image. Left and Middle show images obtained with different staining processes and hence the overall color difference. The right one is the zoomed in view of the middle image showing the different types of axons.

tion of the OR-axons involves taking as input a microscopic image (Figure 1a) and creating a density map (Figure 10c,d) where blue regions indicate high density clustering of OR-axons. The complexity of this automation is compounded by the following facts.

- The microscopic image is littered with a large amount of 'cellular debris', i.e. proteins and other cell bodies that have to be identified and rejected, or not identified at all.

- The oligodendrocyte cells do not conform to any particular shape. In fact, even basic shape properties like convexity cannot be assumed. This demands a shape independent classifier to distinguish the cells.

- The average intensity level of the input images might be different depending on the staining process (Figure 1a and 1b). Hence relating intensities across images for consistently identifying the cells is not possible, and this intensity threshold for cell identification has to be learned within each image.

\subsection{Main Contribution}

Most existing methods for automatic cell analysis focus on large, cellular matrices [19]. However, in cases where the topology of the cellular structures is not uniform, as is typically the case in injury sites, methods for identification and quantification of individual cells must be employed. Our main contribution is in designing an end-to-end automated process that analyzes the input microscopic image and returns an effective high-level visualization of the distribution of the OR-axons. We present a robust algorithm to handle noise, subjectivity and under-sampling, by using efficient computational geometry and stable statistical techniques.Our method consists of the following five key steps.

1. Contour Detection: First, we detect the myelin sheath surrounding the axons using progressive isocontours at varying intensity levels (Section 3). This technique is a shape independent process that makes use of the variational property of the intensities of the cell structures. This also allows us to detect axon boundaries across images of different intensities and pathologies stained by different dyes.

2. Contour Separation: Since the contour detection step does not consider the shape of the axons, the boundaries detected by this step may include multiple axons. We use a Delaunay triangulation based method to separate these cells and define a clean and unique boundary for each of them (Section 4).

3. Noise Removal: The cells detected by the above steps may include noise (e.g. cellular debris). In this step, we identify this noise and remove the cells that do not represent axon structures (Section 5).

4. OR-Axon Classification: G-ratio is defined as the ratio of the cell boundary (myelin sheath) thickness to the cell diameter. The remyelination due to different cells can be differentiated by the G-ratio of the cell. In this step, we design a method based on G-ratio computation and clustering to identify the oligodendrocyte-remyelinated (OR)-axons from other axons (Section 6).

5. Density Map Generation: Finally, we use the distribution of the detected OR-axons in the image to generate a density map that is effective in visualizing the size, location and density of their clusters in the area of the pathology (Section 7).

The pipeline of the entire process, as described above, is illustrated in Figure 2. We corroborate our method by extensive crossverification by the domain experts to demonstrate its accuracy and robustness.

We first discuss the relevant literature in Section 2. Then we present the four steps of our automated algorithm in Sections 3 to 7. This is followed by experiments and analysis in Section 8. Finally, we conclude in Section 9.

\section{RELATED WORK}

Digital image cytometry [21], the analysis of cell attributes from images, serves as an essential component of biological research. Correct detection of relevant cell structure and boundaries is a challenging task in image cytometry because of the varying intensity levels in the images occurring from different staining protocols, varying sizes of the specimen, difference in shapes across cell structures and the presence of large numbers of cellular debris.

Cell detection algorithms using image processing and computer vision techniques continue to be a significant area of research [21, $17,2,20]$. Shape based analysis of cells is a common approach used by researchers [5] and is applicable to types of cells that conform to specific shapes. Since the OR-axons can be of any arbitrary shape, the shape dependent methods are not useful and shape invariant analysis must be used for detection, identification and classification of axons.

Watershed segmentation is a widely used shape-independent approach for cell detection [9], but this algorithm is susceptible to the presence of noise in the image. A single outlier can impact large groups of pixels thereby making the watershed segmentation unstable. Most cell detection algorithms usually detect some cell structures that are merged and need to be separated. Thus, segmentation or separation of cell boundaries to detect individual cells is an active area of research [14, 21]. The active contours method [20, 18, $21]$ is successfully employed for cell analysis and usually produces robust boundaries even for noisy images. Zimmer et al. [21] use an active contour based cell segmentation approach to detect and track motile cells. They use an edge map computed from deviation between local intensities to detect low contrast boundaries. While active contours detects the cell structure by energy minimization subject to external and internal constraints, our proposed approach uses different contours of the same image to classify a structure as a cell by analyzing the evolution of boundaries detected by the isocontour algorithm progressively. 


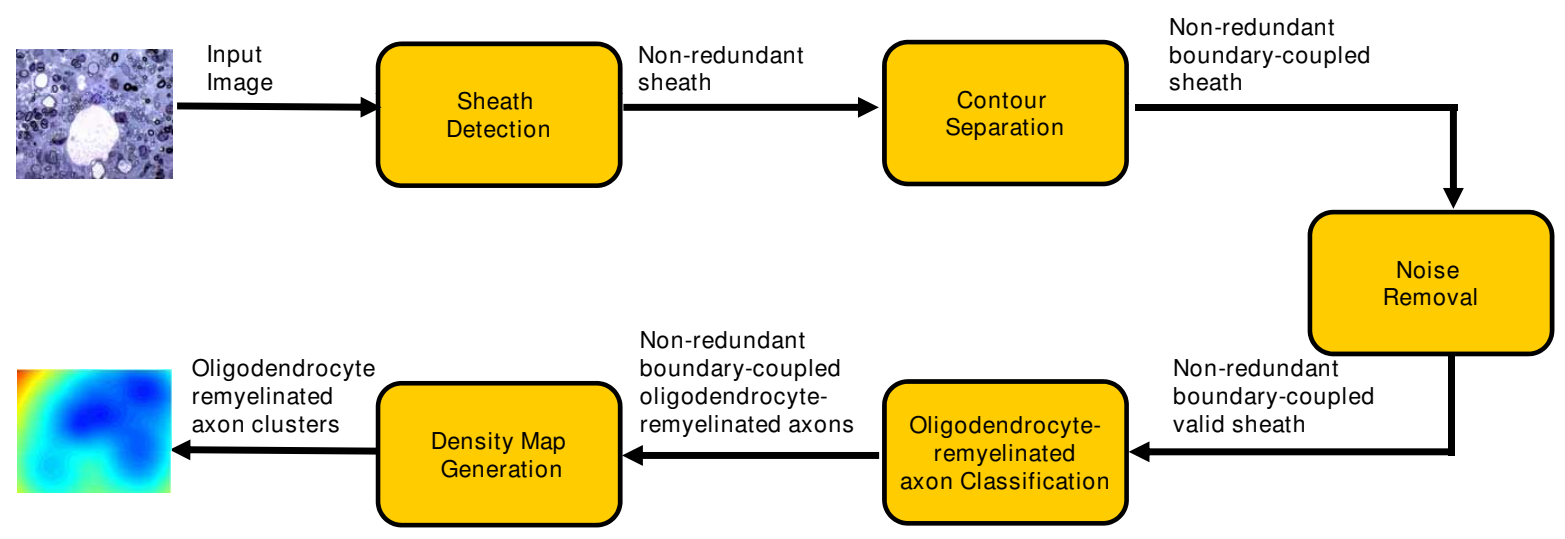

Figure 2: The pipeline of our proposed algorithm.

In almost all applications, the nature of staining makes the nuclei of cells more prominent compared to the outer cell boundaries. A good cell segmentation algorithm has to correctly identify the separating region between the adjacent cell nuclei. Using a known cell nucleus, Jones et al. [10] use non-Euclidean Voronoi diagrams on Riemannian manifolds to detect cell boundaries and segment cells. The cell nucleus is not known in our applications, and hence the approach proposed in [10] is not applicable to our problem. We use an Euclidean-space Delaunay triangulation based approach to separate the detected axon boundary contours obtained from progressive isocontouring. Furthermore, our method directly works on the geometry of the $2 \mathrm{D}$ cell structure provided by the isocontouring algorithm, while [10] works on higher dimensional space derived from the image parameters.

We introduce the concept of progressive isocontouring to detect all the cell structures followed by contour separation using Delaunay triangulation. This allows us to detect event points when there is a morphological change in the shape of the isocontours which can subsequenly be used in the post processing stage to detect generic cell structures. Similar computational geometry-based approaches to topology tracking include efficient implementations of Reeb graphs [6]. ${ }^{1}$ The detected cell structures, once identified as axons, are processed to identify the subset of OR-axons using robust geometrical and statistical methods.

\section{CONTOUR DETECTION}

Traditionally, axon detection algorithms use two techniques shape dependent analysis and edge detection. Since the OR-axons can be of any shape, typical shape matching algorithms cannot be used in our applications. Further, traditional edge detection methods also cannot be used due to the presence of an abnormal number of debris in the image, and the need for correct computation of relative size of the detected boundaries in order to classify the axons.

The OR-axons sometimes can be classified by their lighter intensity values in the image slices. The intensity value at which these axons can be detected can vary over the time elapsed from the remyelination procedure, and also depends on the subjective manual staining process before the microscopic images are taken. Hence isocontouring at one specific intensity level will not be a feasible solution for this problem.

We present the progressive isocontouring technique - isocontouring at progressively increasing intensity levels - as a generic method to detect possible axon structures. For this purpose, we first define the axon structure in this context as illustrated in Figure 3. Let us consider two simple closed iso-contours at intensity $i$ - the ${ }^{1}$ Note that although the analysis of evolving topology and its events
is also the basis of Morse theory [12], its application is limited in
the context of medical image processing due to the complexity of the image content [8].

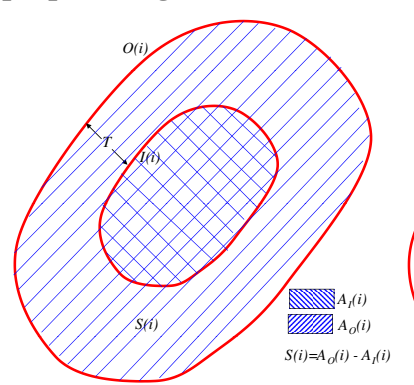

(a)

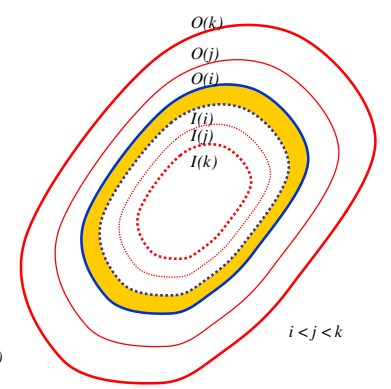

(b)
Figure 3: (a) This schematic illustrates the axon and sheath structures in the image. (b) This illustrates the redundant sheath structures in red. The blue sheath is the one detected at the lowest intensity and hence it is retained as the nonredundant sheath structure.

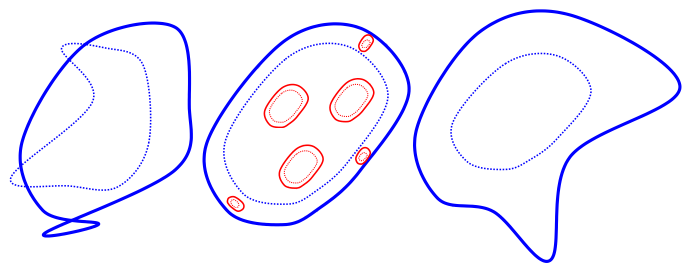

Figure 4: This schematic shows all the invalid axons. Left: Invalid axons due to self-intersecting boundary or intersecting inner and outer boundary; Middle: Invalid axon due to axonwithin-axon structure; Right: Invalid axon due to local discontinuity in sheath thickness.

inner contour $I(i)$ and the outer contour $O(i)$. Let these two enclose regions $A_{I}(i)$ and $A_{O}(i)$ respectively such that $A_{I}(i) \subset A_{O}(i)$. Then $A_{O}(i)$ constitutes an axon and the region between the contours $I(i)$ and $O(i)$, given by $A_{O}(i)-A_{I}(i)$, constitutes the myelin sheath $S(i)$. The thickness $T(i)$ of the sheath $S(i)$ is given by the average of the closest distances of every inner contour point to the outer contour. This is illustrated in Figure 3.

We compute iso-contours progressively at different intensity levels moving from lower to higher intensities. A sheath $S(i)$ detected at intensity level $i$ signifies a valid structure if it obeys the rules described below. These rules are framed based on our observations of the structure of the axon and its surrounding myelin sheath in the images.

1. Boundary Coupling: There exists a one-to-one and onto relationship between the inner and outer boundaries of a sheath structure $S(i)$. This maintains the invariance that every inner boundary has a corresponding unique outer boundary and vice-versa. 
$\rightarrow v^{\circ}$

(a)

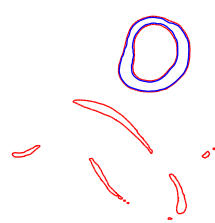

(d)

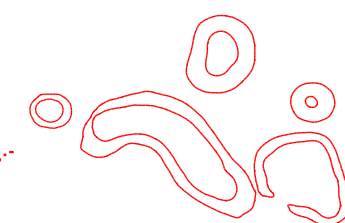

(b)

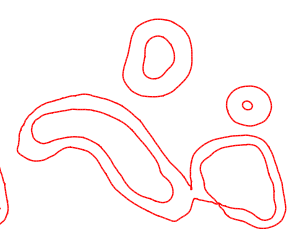

(c)

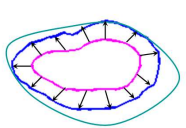

(a)

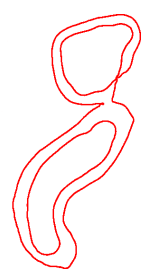

(b)

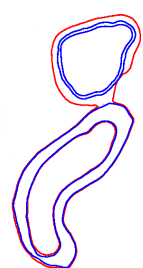

(c)

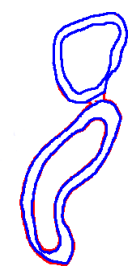

(d)
Figure 6: (a) Contour dilation process on Real Data. (bd)Problems with dilation based axon-separation. (b) The outer contour merges, even as the outer contour of one of the two sheaths has not been detected. (c) One of the inner contours that does not have a corresponding outer contour is dilated to the minimum distance between itself and the merged outer contour. Note the thickness is much smaller than the reasonably correct shape given by the red contour. Such problems will skew the G-ratio of this axon, and hence its classification. (d) Our Delaunay based separation algorithm computes the correct sheath shape.

single outer boundary violating the boundary coupling criterion as shown in Figure 5c. These are handled in the contour separation step, as explained in Section 4. Further, there can also be multiple outer boundaries associated with a single inner boundary violating the valid sheath structure criterion. These are handled in the noise removal step, as explained in Section 5.

The isocontours at specific intensity levels show the regions of the axon in different topologies: the axon boundaries might have multiple components (Figure 5a) or multiple axons might be merged into a single axon boundary (Figure 5c). On the other hand an analysis of the change in topology of the axon boundaries over the isocontours of smoothly varying intensity levels provides a wealth of information for identifying the sheath structures accurately. This is the key observation behind the progressive isocontouring method. We compute the curves with the same brightness (isocontours) in the image repeatedly for progressively increasing intensity values (Figure 5). When the topology of the isocontour changes over subsequent higher intensity levels, we analyze it to detect the formation of new sheath structures (two closed curves one containing the other). These sheaths identified at a specific intensity level (blue curves in Figure 5d-f), would be enclosed by the isocontours in the subsequent intensity level images (red curves in Figure 5). These containments are detected and only the blue isocontours (with smaller sheath thickness) are retained as a non-redundant sheath structure.

\section{CONTOUR SEPARATION}

After the first step of the pipeline we get non-redundant sheaths, but they may not satisfy the boundary coupling criterion. There can be detected outer boundary contours that enclose multiple smaller inner boundary contours. This is due to the fact that any pathology being imaged by the microsope, though very thin, still has a nonzero thickness. Each cell seen in the image will have a slightly different depth than the others. Though their mobility is restricted, they can sometimes occlude parts of other cells merging visible outer boundaries.

Figure 7a illustrates the typical scenario of merged contours where the outer boundary of multiple myelin sheath structures are merged. This step of our algorithm divides the outer boundary into required numbers of closed curves to match with each enclosed inner boundary (Figure 7d).

One simpler and obvious solution to separate the contours is by dilating the inner contour along the normal direction by the min- 


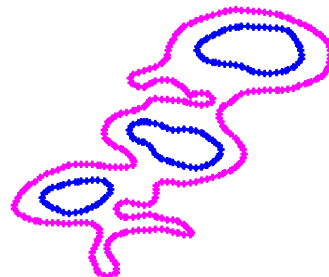

(a)

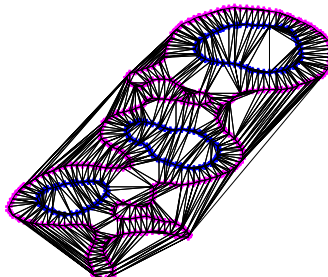

(b)

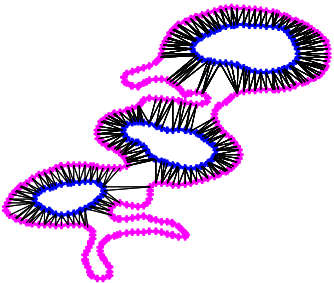

(c)

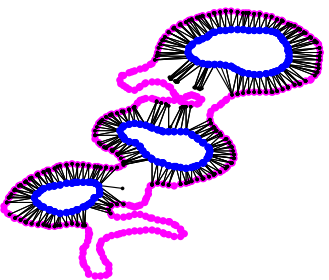

(d)

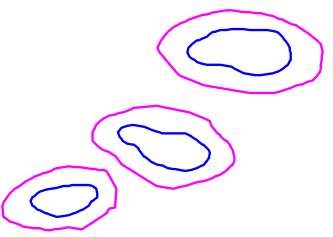

(e)

Figure 7: Contour separation algorithm on Real Data: (a) The pink outer boundary encloses multiple blue inner boundaries violating the boundary coupling criterion. (b) The Delaunay triangulation of the vertices on inner and outer boundary contours. (b) The edges of the triangulation that connect any inner boundary vertex to an outer boundary vertex are retained. (d) The edges incident on each inner boundary that are statistical outliers with respect to edge length are shortened to match the average edge length of these edges. (e) The closed loop of vertices thus formed by the farther ends of the edges incident on each boundary forms the corresponding outer boundary thus separating the merged outer boundary contour.

imum distance to the outer contour enclosing it (Figure 6a). Although this method works reasonably well in many cases, it is not robust and is susceptible to noise (cellular debris) in the image. It may lead to sheath structures with inaccurate thicknesses and hence inaccurate G-ratio value and eventual misclassification of the ORaxons (Figure 6c).

To avoid these problems, we propose a robust Delaunay triangulation based contour separation algorithm. A triangulation of a point set is Delaunay if no point is inside the circumcircle of any triangle of the triangulation. Hence, a point is always connected to its closest point in Delaunay triangulation. Before detailing the algorithm, let us consider a sheath whose inner and outer boundaries, $I$ and $O$ are detected and coupled correctly. Let us consider a Delaunay triangulation of the vertices in the two boundaries, i.e. $I \cup O$. Let us retain only those edges from this triangulation that connect a point in the inner boundary to a point in the outer boundary. This retained set of edges corresponds to the sheath $S$ in which all edges have lengths close to the thickness of the sheath (Figure 7). Further, we also notice empirically that all vertices in the outer contour are connected to at least one inner contour vertex. We use these observations to handle situations where there are multiple inner contours within a single outer contour (Figure 7a).

The contour separation method takes as input $N, N>1$, inner contours, denoted by $I_{k}, 1 \leq k \leq N$ and their single outer contour $O$. The output is $N$ closed outer contours, $O_{k}$ one corresponding to each inner contour $I_{k}$, carved out of $O$. The basic observation is that around each inner contour $I_{k}$, the outer contour $O$ is well formed except in areas of concavities in $O$ (Figure 7a). For this we first perform a Delaunay triangulation of all the points in $I_{k}, 1 \leq k \leq N$, and $O$ (Figure $7 \mathrm{~b}$ ). Next, we retain the edges connecting the inner boundary vertices to the outer boundary vertices (Figure 7c). Note that the Delaunay edges in the concavities of $O$ connect two outer boundary vertices and are hence removed.

As discussed earlier, in an ideal cell with coupled inner and outer boundary contours, the retained Delaunay edges incident on the inner boundary vertices are all of similar lengths. So, we find the edges incident on each $I_{k}$ that are statistical outliers in terms of their length. These are usually the edges which couple an inner boundary vertex to an incorrect vertex in the outer boundary or a different inner boundary. If an outer boundary vertex $p_{o}$ is connected only by these retained Delaunay edges that are statistical outliers then each of these edges connected to $p_{o}$ is shortened away from $p_{o}$ to the average length of all the other non-outlier edges incident on $I_{k}$ (Figure 7d). The outer boundary $O_{k}$ is now formed by connecting the farther end points of the edges incident on $I_{k}$ in order (Figure 7e).

This Delaunay based contour separation method is extremely robust. This algorithm is also provably correct as it retains only those Delaunay triangles whose circumcenter is close to the medial axis [1] formed by the coupled inner-outer contour pair points, thus es-
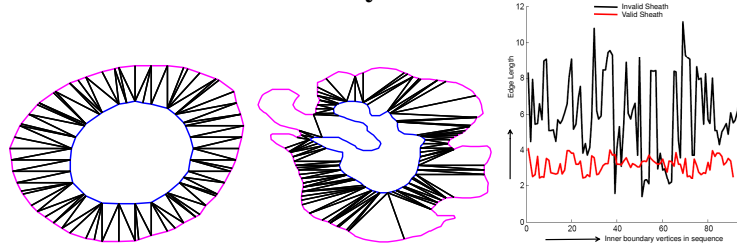

Figure 8: This figure illustrates the local discontinuities in edge length in an invalid sheath when compared to a valid sheath in real data. The left and the center images show a valid and an invalid sheath respectively. The right image shows the plot of the sheath thickness as we move along the contour of the valid and invalid sheath. The valid sheath shows a locally smooth plot while the bad cell shows severe local discontinuities.

tablishing the one-to-one and onto correspondence between the inner and outer contours as prescribed by the boundary coupling criteria for the definition of a sheath structure. Further the lengths of the edges retained in this Delaunay triangulation provide a very good estimate of sheath thickness which is later used in our G-ratio computation and classification (Section 6).

\section{NOISE REMOVAL}

Following the contour separation step, we get non-redundant boundary coupled sheath structures, but we still have many invalid sheath structures detected due to cellular debris. In this step we identify such cellular debris and remove them. After the removal of cellular debris, the remaining cell structures satisfy all the rules of a valid axon structure described in Section 3.

Sheath structures that have self-intersecting boundaries and intersecting inner and outer boundaries can be identified using simple line-polygon intersection algorithms and removed. The invalidity due to axon-within-axon (Figure 4) is detected using the same contour within contour test as used to differentiate inner boundaries from outer boundaries in Section 3. Note that axon-within-axon noise structures are different from structures that require axon separation. The former structure has multiple axons (with both inner and outer contours) inside another axon (again with both inner and outer contours), while the latter has multiple inner contours inside a single outer contour.

The last case of invalid sheath structure is one with locally nonuniform sheath thickness. This is detected using an algorithm similar to the one proposed for contour-separation. We retain the Delaunay edges of the vertices in the inner $(I)$ and outer $(O)$ contours. It can be clearly seen from a typical example in Figure $8 \mathrm{c}$ that the variance in the edge lengths of the valid and invalid sheaths are clearly different and can be used to distinguish one from the other.

\section{OLIGODENDROCYTE-REMYELINATED AXON CLASSIFICATION}

The identification and classification of oligidendrocyte-remyelinated axons are done based on a parameter called G-ratio. The G-ratio is 


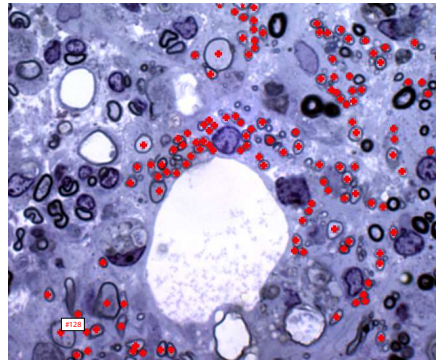

(a)

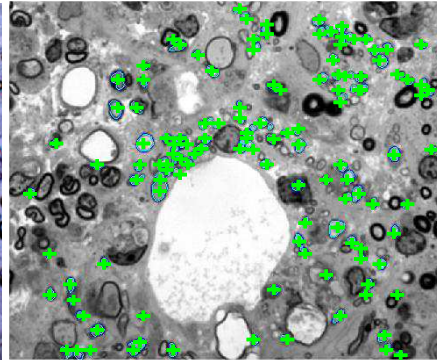

(b)

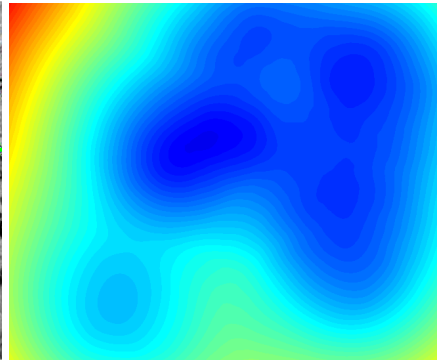

(c)

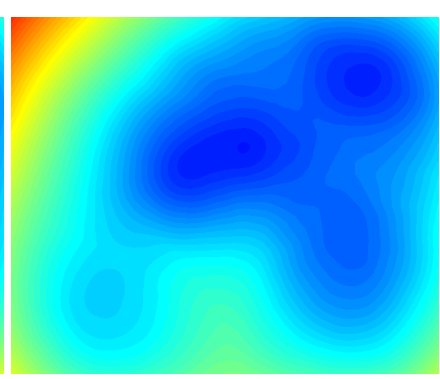

(d)

Figure 10: (a) Image shows the manual count of OR-axons represented using red markers. (b) Automatic count of oligodendrocyteremyelinated axons on the same image, using the algorithm presented in this paper. (c) Visualization of the cluster density map of the manual count (d) Density map of the automated count. Images (c) and (d) should be similar if the actual and the manual counts closely match. Using image similarity measure SSIM [16], we show that the two density images are indeed very similar (SSIM $=0.982$ ).

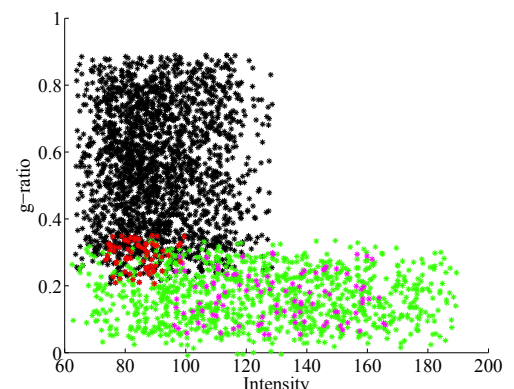

Figure 9: K-means clustering algorithm for Axon Classification: The Y-axis shows the G-ratio of the axons. The $\mathrm{X}$-axis is the intensity level at which specific axons were detected using the progressive isocontour process. The greeb points represent the OR-axons (true positives), the magenta, red and black points represent the false positives, false negatives and true negatives respectively. The data is accumulated over many microscopic images spanning different staining intensities, and with different numbers of axons.

defined as the ratio of the sheath thickness to the axon diameter. To find the sheath thickness, we perform the Delaunay triangulation of its inner and outer boundary vertices and retain only the edges that connect the inner boundary vertices to the outer boundary vertices. The mean length of these retained edges approximates the sheath thickness. The axon diameter is computed as the largest distance between any two points in the inner boundary $I$.

There are three kinds of myelinated axons: (a) normally myelinated axons are those that were not affected during the spinal cord injury, (b) the axons that were remyelinated by the Schwann cells and (c) the axons that were remyelinated by the oligodendrocyte cells that were injected in the course of therapy. Our goal is to detect and count the OR-axons in order to monitor the progress of oligodendrocyte based remyelination which is a critical step in estimating the efficacy of the therapy. Oligodendrocyte remyelinated axons are characterized by lower G-ratios than the normally or Schwann remyelinated axons. Hence, to classify these therapeutically important axons we perform k-means clustering of the G-ratios with number of clusters set to two. The axons corresponding to the cluster with lower G-ratio are the OR-axons.

Since, typically during manual counting, it is prohibitively tedious to measure G-ratios for all the detected sheaths, the conventional way to identify an OR-axon is by the lighter intensity of its myelin sheath when compared to the darker, more compact, Schwann cell-remyelinated axons $[15,7]$. On the other hand, since we can accurately measure G-ratios automatically, we use relative G-ratios for clustering the OR-axons. However, we make an interesting observation from this classification. While it is true that all cells with lighter intensity sheaths are OR-axons, the converse is not true - not all OR-axons have lighter intensity sheaths. Hence, intensity based manual classification is prone to errors. Further, the intensity levels at which the axons are detected change based on the staining process before the microscopic images are taken. This makes intensity based manual classification subjective. Our automated G-ratio based classification thus reduces classification errors significantly.

The results of this clustering method for several images are shown in Figure 9. In this figure we plot the G-ratio of the axons (Y-axis) against the intensity levels (X-axis) of the progressive isocontouring algorithm at which the axon was detected. The image shows the plot of cells in over 15 images with variable staining intensities and different numbers of recognizable cells. Aggregated statistics from all these images are as follows: all the cells recognized at an intensity level above 130 are oligodendrocyte remyelinated axons so are all the cells with G-ratio below 0.2. Further, all the cells with G-ratio above 0.35 are non-oligodendrocyte myelinated axons. The region of confusion is in the rectangular range of intensity less than 130 and the G-ratio between 0.2 and 0.35 . The green points in the figure denote the OR-axons and the black points mark all other cells. The false positives and false negatives are shown in magenta and red respectively. Further analysis of these results is given in Section 8 .

\section{DENSITY MAP GENERATION}

To evaluate the effectiveness of the remyelination therapy it is important to identify the regions of the image where large clusters of high density OR-axons occur. The size and density of these clusters indicate the rate of progress and the location indicates the appropriateness of the targeted cite of the therapy.

To facilitate this process we calculate a density map as follows: the centroids of all the detected OR-axons are identified, and for each pixel in the image, the average distance to its $k$ nearest centroids are calculated. Color coding of these values gives the density image that visually represents the relative concentration of the detected cells. Lower values indicate higher concentration of ORaxons and vice versa. Hence, cooler colors (blue) indicate the cites of remyelination due to oligodendrocytes and warmer colors (yellow and red) indicate lack thereoff. Figure 10 shows the density maps thus calculated for the corresponding input images shown in the same Figure.

\section{RESULTS AND ANALYSIS}

We used Sprague Dawley female adult rats with 200 KiloDyne force spinal cord contusion injury. All tissues were fixed and processed as described in [15]. The images of these tissues were used as input to our system. The implementation of the algorithm was prototyped using MATLAB and the images used had different intensities. The shapes and scales of the cells also varied across im- 
ages. The remyelination analysis that we automated was also carried on manually by experts without the knowledge of the results of the automated process. The experts were also consulted to review the results of the automated process.

Sample classification results with manual count and automatic counts are demonstrated in Figure 12. The left column of Figure 12 denotes the greyscale images with manual counts marked with red and the right column denotes the results from our automatic classification with the detected cells marked in green. Figure 11 shows another example of our automatic classification. False negatives are denoted by red and false positives by magenta.

Additional results of the manual and automatic counts are shown in Table 1. Although manual counting by experts suffers from classification error due to the subjective nature of the task, all the statistics and analysis has been done assuming that the expert counting for the first time (before seeing the results of the automated process) is the ground truth (Column 1 of Table 1). The same images were then processed using our method and the automatic count of OR-axons were reported in Table 1. Correctly classified OR-axons were considered to be true positives (denoted by green in Figure 9), and the cells that are chosen by the system but not by the experts are false positive (denoted by magenta in Figure 9).

\begin{tabular}{|l|l|l|l|}
\hline $\begin{array}{l}\text { Actual count of } \\
\text { axons }\end{array}$ & $\begin{array}{l}\text { Automated } \\
\text { count of axons }\end{array}$ & Hit rate & $\begin{array}{l}\text { Excess } \\
\text { rate }\end{array}$ \\
\hline 68 & 65 & 94.12 & 1.4 \\
\hline 78 & 80 & 92.31 & 10.2 \\
\hline 96 & 100 & 91.67 & 12.5 \\
\hline 35 & 38 & 94.29 & 14.28 \\
\hline 25 & 30 & 96 & 24 \\
\hline
\end{tabular}

Table 1: Empirical performance evaluation using domain expert's count as ground truth: OR-axons detected by our algorithm closely match the ground truth, demonstrated by the high hit rate.

Traditionally, the success of an automated method is defined by the hit rate given by

$$
\text { hit rate }=\frac{\text { Number of true positives }}{\text { Manual count of OR axons }} .
$$

This is usually accompanied by a false alarm rate given by

$$
\text { false alarm rate }=\frac{\text { Number of false positives }}{\text { Manual count of non-OR axons }} .
$$

However, since the non-OR axons are of no importance in the context of remyelination, manual count for non-OR cells are not available for us to calculate false alarm rate. Hence, we define a new term called excess rate as

$$
\text { excess rate }=\frac{\text { Number of false positives }}{\text { Manual count of OR axons }} .
$$

A high hit rate signifies effective classification of OR axons while a low excess rate signifies effective rejection of non-OR axons. Hence, the hit rate together with the excess rate provides an important indicator for the accuracy of the automated remyelination analysis. A high hit-rate ( $>90 \%)$ accompanied by a low excess rate $(<25 \%)$ signifies a successful automated analysis.

The false positives (shown in magenta in Figure 9) either have high intensity or have very low G-ratio values indicating that they are indeed within the statistical range of correct OR-axons. Interestingly, on showing the results of the automatic cell detection, the experts concurred that over $95 \%$ of false positives (cells chosen by us but not the experts) were actually true positives. These true positives were missed due to several human factors like subjectivity and fatigue. This demonstrates the need for automated analysis and also validates a higher accuracy for our automated analysis by increasing the hit rates and reducing the excess rates.

On the other hand, the false negatives (shown in red) which determines the miss rate (i.e. 1-hit rate), in Figure 9, is in the boundary of true positives (shown in green) and true negatives (shown in black) and hence are difficult to automatically label them correctly using only G-ratio and intensity. A similar pattern is observed when considering single images, as shown in Figure 11. Interestingly, classification of the false negatives was also subjective among human experts and the misclassification by the automated system is well within the variance experienced in human counting.

The most important validation of the success of the automated analysis would be in comparing a density map generated from the clusters created by the automated process with the one that is created from the clusters marked by the manual process since this is the visualization that is ultimately used for detecting the growth and site of remyelination. Figure 10 shows an example of density images for both manual counts and automatic counts for a sample image. The number of $k$ nearest neighbors was fixed at $1 / 4^{\text {th }}$ of the number of cells in the manual count. Blue regions in the density image denote areas having higher density of detected cells (Figures 10c and 10d).

In order to quantify the image similarity between the density images from the manual and automated counts, we use a well established image similarity measure, Structural SIMilarity (SSIM) index [16]. SSIM is an objective method for assessing perceptual image quality using structural similarity between images. The mean SSIM index value of 1.0 between two images denotes two identical images. For the image shown in Figure 10, the mean SSIM index value between the density images corresponding to manual and automated count is 0.982 . The mean SSIM index for all the images reported in Table 1 is $0.97( \pm 0.02)$. Thus the method not only counts the desired cells with high degree of accuracy, but also identifies, classifies, and labels individual axons with very high confidence.

\section{CONCLUSION}

We have presented an end-to-end process for accurate analysis and visualization of the clusters of OR-axons critical to evaluate the progress of remyelination therapy. Our automated cell classification always correctly identifies the region populated by the OR-axons, and closely matches the manual classification. Furthermore, the automated identification has a natural advantage of objectively classifying the axons. This automated remyelination analysis and visualization has also injected a lot of excitement among our neurobiologist collaborators. This project will relieve them of several weeks of a pain-staking, repetitive, and mundane task that consumes several hours of trained manpower. By virtue of its accuracy in detection and classification of axons, we hope this method will find widespread applicability [11] thus reducing the turnaround time of the neurobiology research.

Though specific in application, there are several components of the algorithm that are generic and can be applied in different types of medical image analysis and visualization. The progressive isocontouring algorithm is a simple and generic image processing technique that can detect closed shapes in general stained microscopic images. In addition, this method also detects event points when the shape topology changes. Any application specific post-processing method can process just these events to detect the required cells. Our Delaunay triangulation based geometric processing contour separation and noise removal methods might evoke great interest in the newly developing bio-geometry community. 


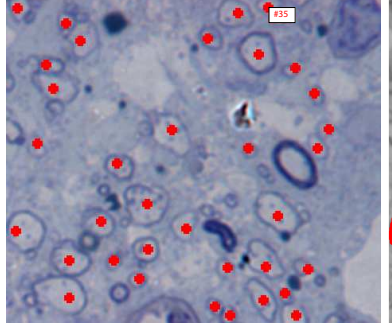

(a)

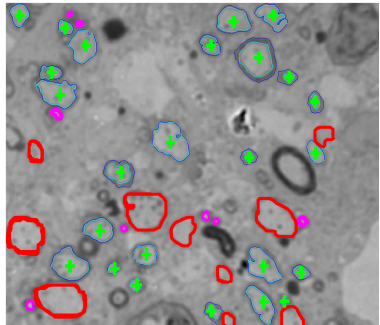

(b)

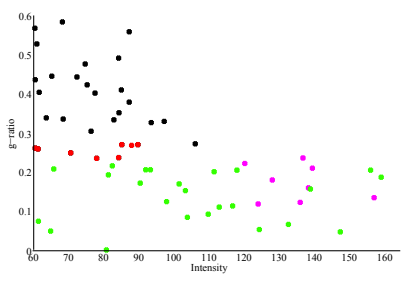

(c)

Figure 11: (a) Manual classification (b) Automatic classification: False positives are shown in magenta, false negatives in red and true positives with green markers as compared to image (a). (c) Shows the k-mean classification of the cells. Note that the false negatives are in the boundary of true positives and true negatives and hence are difficult to automatically label them correctly using only G-ratio and intensity classifiers.

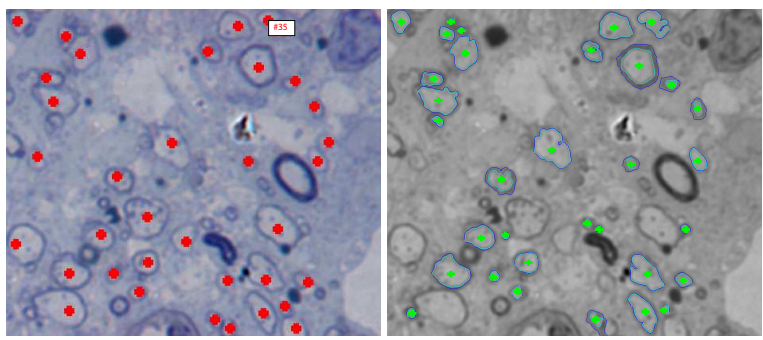

(a) ( manual: 35; automated: 33)
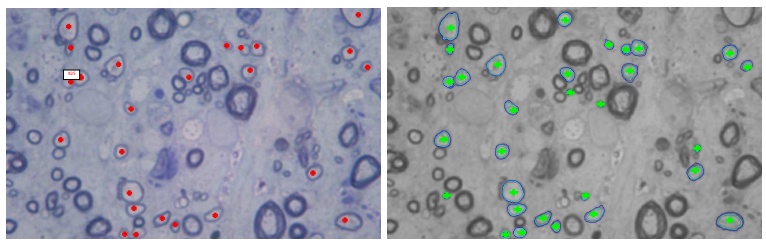

(b) (manual: 25; automated: 30)

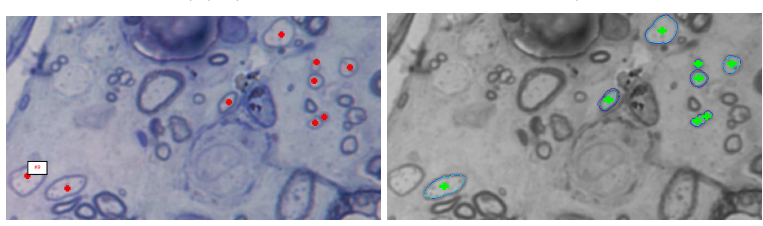

(c) ( manual: 9; automated: 8)

Figure 12: Classification results: Left: Stained images showing manual classification by domain experts; Right: Gray images showing classification using our automated method. The caption shows the number of OR-axons classified manually and automatically. Note that the domain experts nor the automated algorithm were aware of each others classification results. Please zoom in to see results.

\section{REFERENCES}

[1] N. Amenta, M. Bern, and D. Eppstein. The crust and the $\beta$-skeleton: Combinatorial curve reconstruction. Graphical Models and Image Processing, 60(2):125 - 135, 1998.

[2] J. Angulo and G. Flandrin. Automated detection of working area of peripheral blood smears using mathematical morphology. Anal Cell Pathol, 25(1):37-49, 2003.

[3] W. Blakemore and H. Keirstead. The origin of remyelinating cells in the central nervous system. J Neuroimmunol, 98:69-76, 1999.

[4] A. Blight. Cellular Morphology of Chronic Spinal Cord Injury in the Cat: Analysis of Myelinated Axons by Line-Sampling. Neuroscience, 10:521-543, 1983.

[5] C. Daul, P. Graebling, and E. Hirsch. From the hough transform to a new approach for the detection and approximation of elliptical arcs. CVIU, 72:215-236, 1998.

[6] H. Doraiswamy and V. Natarajan. Efficient algorithms for computing reeb graphs. Comput. Geom. Theory Appl.,
42(6-7):606-616, 2009.

[7] J. Guy, E. A. Ellis, K. Kelley, and G. M. Hope. Spectra of G-ratio, myelin sheath thickness, and axon and fiber diameter in the guinea pig optic nerve. J Comp Neurol, 287:446-454, 1989.

[8] A. Gyulassy, P.-T. Bremer, B. Hamann, and V. Pascucci. A practical approach to morse-smale complex computation: Scalability and generality. IEEE TVCG, 14, 2008.

[9] D. Hagyard, M. Razaz, and P. Atkin. Analysis of watershed algorithms for greyscale images. In ICIP, pages III: 41-44, 1996.

[10] T. Jones, A. Carpenter, and P. Golland. Voronoi-based segmentation of cells on image manifolds. ICPR, 2:286-289, 2002.

[11] J. Meyer, K. Velasco, and M. Gopi. Tracking of oligodendrocyte remyelinated axons in spinal cords. In AIChE(Poster), 2008.

[12] J. Milnor. Morse Theory. Princeton University Press, 1969.

[13] J. Prineas. Pathology of the early lesion in multiple sclerosis. Hum Pathol, 6:531-534, 1975.

[14] B. S. Watershed, hierarchical segmentation and waterfall algorithm. In Mathematical Morphology and its Applications to Image Processing, pages 69-76, 1994.

[15] M. Totoiu and H. Keirstead. Spinal cord injury is accompanied by chronic progressive demyelination. J Comp Neurol, 486(373-383), 2005.

[16] Z. Wang, A. C. Bovik, H. R. Sheikh, and E. P. Simoncelli. Image quality assessment: From error visibility to structural similarity. IEEE Transactions on Image Processing, 13(4):600-612, 2004.

[17] D. Wu and Q. Zhang. A novel approach for cell segmentation based on directional information. In ICBBE 2007, pages 920-923, July 2007.

[18] C. Xu and J. L. Prince. Snakes, shapes, and gradient vector flow. IEEE Transactions on Image Processing, 7(3):359-369, 1998.

[19] X. Zhou, X. Cao, Z. Perlman, and S. T. C. Wong. A computerized cellular imaging system for high content analysis in monastrol suppressor screens. J. of Biomedical Informatics, 39(2):115-125, 2006.

[20] C. Zimmer, E. Labruyere, V. Meas-Yedid, N. Guillen, and J.-C. Olivo-Marin. Segmentation and tracking of migrating cells in videomicroscopy with parametric active contours: a tool for cell-based drug testing. IEEE. J. Med. Imaging, 21(10):1212-1221, Oct. 2002.

[21] C. Zimmer, E. Labruyere, V. Meas-Yedid, N. Guillen, and J.-C. Olivo-Marin. Improving active contours for segmentation and tracking of motile cells in videomicroscopy. Computer Vision for Biomedical Image Applications(poster), 3765, 2005. 